\title{
Abnormal expression of p27kip I protein in levator ani muscle of aging women with pelvic floor disorders - a relationship to the cellular differentiation and degeneration Antonin Bukovsky*1,2, Pleas Copas ${ }^{2}$, Michael R Caudle,2, Maria Cekanova ${ }^{1}$, Tamara Dassanayake ${ }^{2}$, Bridgett Asbury ${ }^{2}$, Stuart E Van Meter3, Robert F Elder ${ }^{2}$, Jeffrey B Brown ${ }^{2}$ and Stephanie B Cross ${ }^{2}$
}

Address: ${ }^{1}$ Laboratory for Development, Differentiation and Cancer, University of Tennessee, Knoxville, Tennessee, USA, ${ }^{2}$ Department of Obstetrics and Gynecology, University of Tennessee, Graduate school of Medicine, Knoxville, Tennessee, USA and 3Department of Pathology, The University of Tennessee Graduate School of Medicine, 1924 Alcoa Highway, Knoxville, USA

E-mail: Antonin Bukovsky* - buko@utk.edu; Pleas Copas - pcopas@utk.edu; Michael R Caudle - mcaudle@mc.utmck.edu; Maria Cekanova - mcekanov@utk.edu; Tamara Dassanayake - tamarad@nashville.net; Bridgett Asbury - bridgettasbury@hotmail.com; Stuart E Van Meter - svanmete@mc.utmck.edu; Robert F Elder - relder@mc.utmck.edu; Jeffrey B Brown - jbrown26@utk.edu; Stephanie B Cross - stephcrossll@home.com

${ }^{*}$ Corresponding author

This article is available from: http://www.biomedcentral.com//472-6890///4

(C) 200 I Bukovsky et al; licensee BioMed Central Ltd. Verbatim copying and redistribution of this article are permitted in any medium for any noncommercial purpose, provided this notice is preserved along with the article's original URL. For commercial use, contact info@biomedcentral.com

\begin{abstract}
Background: Pelvic floor disorders affect almost $50 \%$ of aging women. An important role in the pelvic floor support belongs to the levator ani muscle. The p27/kip I (p27) protein, multifunctional cyclin-dependent kinase inhibitor, shows changing expression in differentiating skeletal muscle cells during development, and relatively high levels of p27 RNA were detected in the normal human skeletal muscles.

Methods: Biopsy samples of levator ani muscle were obtained from 22 symptomatic patients with stress urinary incontinence, pelvic organ prolapse, and overlaps (age range 38-74), and nine asymptomatic women (age $3 \mathrm{I}-49$ ). Cryostat sections were investigated for $\mathrm{p} 27$ protein expression and type I (slow twitch) and type II (fast twitch) fibers.

Results: All fibers exhibited strong plasma membrane (and nuclear) p27 protein expression. cytoplasmic p27 expression was virtually absent in asymptomatic women. In perimenopausal symptomatic patients (ages 38-55), muscle fibers showed hypertrophy and moderate cytoplasmic p27 staining accompanied by diminution of type Il fibers. Older symptomatic patients (ages 57-74) showed cytoplasmic p27 overexpression accompanied by shrinking, cytoplasmic vacuolization and fragmentation of muscle cells. The plasma membrane and cytoplasmic p27 expression was not unique to the muscle cells. Under certain circumstances, it was also detected in other cell types (epithelium of ectocervix and luteal cells).

Conclusions: This is the first report on the unusual (plasma membrane and cytoplasmic) expression of p27 protein in normal and abnormal human striated muscle cells in vivo. Our data indicate that pelvic floor disorders are in perimenopausal patients associated with an appearance of moderate cytoplasmic p27 expression, accompanying hypertrophy and transition of type II into type I fibers. The patients in advanced postmenopause show shrinking and fragmentation of muscle fibers associated with strong cytoplasmic p27 expression.
\end{abstract}




\section{Background}

Pelvic floor disorders (PFD) are highly prevalent among elderly women. Many surgical reports attempt to determine the best surgical treatment for PFD, yet do not address the pathophysiology. The etiology of PFD is probably multifactorial, including a genetic predisposition to connective tissue abnormalities, vaginal childbirth with damage to the innervation of the pelvic floor muscles, estrogen deficiency, and aging effects [1]. The pelvic floor, situated at the bottom of the abdominal cavity, forms a supporting shelf for the abdominal and pelvic viscera. It has three layers: the endopelvic fascia, the levator fasciae and muscles, and the perineal membrane/ external anal sphincter.

The primary support for the pelvic organs comes from the pelvic floor muscles $[1,2]$. This suggests that an alteration to the levator ani supportive function may significantly contribute to the pathogenesis of PFD [3], which affect $5 \%$ of younger and nearly $50 \%$ of elderly women [2]. In women, the levator muscles form a horizontal shelf with an anterior midline cleft (the urogenital hiatus) through which the urethra, vagina, and rectum pass. Anatomically, the levator muscle is subdivided into the iliococcygeal and the pubococcygeal muscles [4,5] (Fig. 1).

The levator ani muscles play an important role in bladder neck fixation provided by the suspensory sling and hiatal ligament [6]. The urethra is supported by the action of the levator ani muscles through their connection to the endopelvic fascia of the anterior vaginal wall [7], and the muscles of the levator ani contract during a cough to assist continence [8]. The fast twitch type II fibers in the levator ani muscles play an important role in continence, and their loss accompanies the development of urinary incontinence [9]. Clinically, women with recurrent uri-

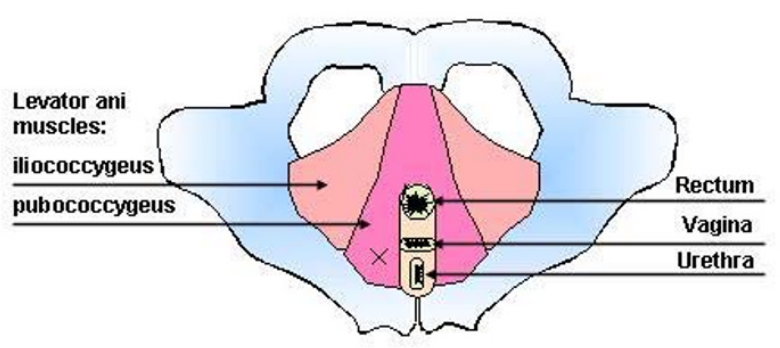

Figure I

Schematic drawing of the pelvic floor. $X$ indicates site of biopsy. nary incontinence after Burch colposuspension have a more pronounced pelvic floor weakness than women with primary stress urinary incontinence [10]. This suggests that the recurrent urinary incontinence is caused by a progression of pelvic floor dysfunction. Severe alteration in the levator ani integrity is associated also with fecal incontinence [11-13].

Histologic and histochemical analysis of pubococcygeal muscle obtained from asymptomatic women and from women with PFD has shown that both age and parity (vaginal delivery) appeared to be related to the morphological features of the samples. In the symptomatic women there was a significant increase in the number of muscle fibers showing pathological damage. The range of diameters of both type I and II fibers was significantly different between symptomatic and asymptomatic women. A significant proportion (90-100\%) of muscle fibers in symptomatic women were type I fibers, showed centrally located nuclei, and exhibited significantly greater diameter than type I fibers in asymptomatic women [3].

However, the cellular and molecular aspects of regression of type II fibers are poorly understood. Though apoptosis in adult muscles has been described, and may result from changes within bcl2/bax system, alteration in pathways that regulate differentiation of muscle cells, changes in the myonuclei, and the nature of pathways inducing senescence of skeletal muscles are still far from being clear [14].

Among cell cycle related proteins, an important role in the regulation of skeletal muscle differentiation belongs

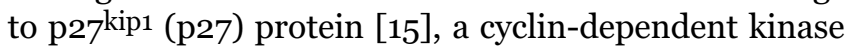
inhibitor required for cell cycle arrest. Additionally, since the identification and cloning of the p27 gene in 1994 [16], the p27 protein has been shown to be involved in various other aspects of cell physiology and pathology [17]. Our previous study has shown, that differentiation of cells in tissue culture is associated with an increase in p27 expression [18]. Although a regular p27 expression protects normal cells from apoptosis, overexpression of p27 induces apoptosis of cells, through elevation of proapoptotic proteins, such as Bax $[19,20]$. These observations indicate that changes in p27 expression can determine the fate of muscle cells.

Aims of the present study were to investigate p27 expression in levator ani muscle of symptomatic women, determine changes with age, and compare the p27 expression with histochemical detection of fast- and slow twitch muscle fibers in parallel sections. 


\section{Materials and Methods Patients}

Biopsy samples $5 \times 5 \times 5 \mathrm{~mm}$ were collected from 22 symptomatic patients with PFD (stress urinary incontinence, pelvic organ prolapse, fecal incontinence, and overlaps; age range 38-74) without hormone replacement therapy. Besides hormone replacement, also excluded from the study were patients with a history of malignancy, endometriosis, and pelvic infections.

Tissue sampling from levator ani muscles and fascia, illustrated in Fig. 1, was based on the work of Bernstein [2] (biopsy size and site), with some modifications approved by the Institutional Review Board. We used similar approaches to obtain the biopsy during reconstructive surgery (symptomatic patients) and abdominal hysterectomy (asymptomatic controls). The biopsy sample was always obtained from the right pubococcygeal muscle. The biopsy was lateral $(20-25 \mathrm{~mm})$ to the urethrovesical junction and was performed midway between the urethra and arcus tendinus (white line). It was lateral enough from the urethra to avoid any alteration of nerves to the urethra and bladder.

In reconstructive surgery, on entering the space of Retzius, the fat was pushed aside to expose the superior fascia of the levator ani. After incision, the fascia biopsy specimen $(5 \times 5 \mathrm{~mm})$ was collected. Next, the Allis clamp was used to expose a small part of the pubococcygeal muscle and collect a $5 \times 5 \times 5 \mathrm{~mm}$ muscle biopsy specimen.
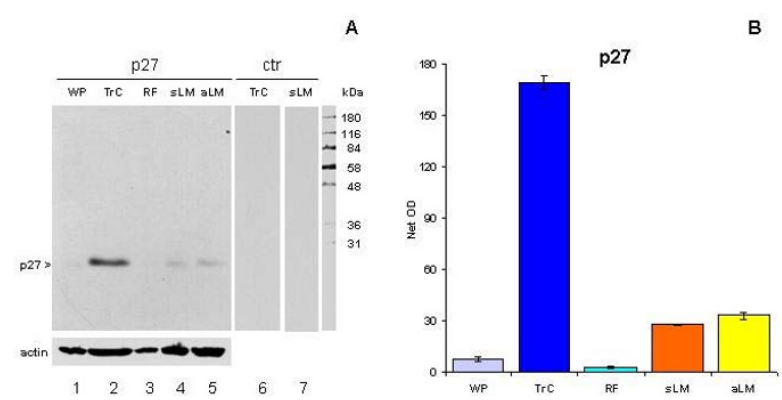

Figure 2

A) Western blot of lysates from whole placenta (WP), trophoblast culture ( $\operatorname{TrC})$, rectus fascia (RF), and levator ani muscle of the symptomatic (sLM) and asymptomatic woman (aLM). Note a single band detected by p27 monoclonal antibody (lanes I-5) and a lack of nonspecific staining (lanes 6 and 7). B) Quantitative evaluation of panel A showed significant differences in the density of p27 expression between all measurements $(P<0.00 \mathrm{I})$, but not WP vs. $\operatorname{RF}(P>0.05)$ and sLM vs. aLM $(P>0.05)$.
For comparison, biopsies were obtained from nine asymptomatic women (age 31-49) who were having a hysterectomy for benign conditions such as abnormal uterine bleeding, fibroids or pelvic pain, and no hormone replacement therapy. They did not have any demonstrable pelvic relaxation by examination or history of stress urinary incontinence. We were unable, during a two year period, collect biopsies from asymptomatic women of age 50 or older. The apparent reason for this is that, except for urogynecological indications and cancer, there is virtually no reason for abdominal gynecologic surgery in postmenopausal women. To ensure the compatibility of biopsy samples, the space of Retzius was opened in all patients.

During abdominal hysterectomy, the urinary bladder was pushed down to reach the space of Retzius by incision right behind the pubic bone. The fat was pushed aside with the assistance of a hand in the vagina, and biopsy was performed as described. After biopsy, a single figure-of-eight stitch suture was placed in all patients, which corrected any potential loss of support that might be caused by the biopsy. This was bloodless and did not create any lasting difficulties. None of the control patients developed urinary incontinence or evidence of loss of support or voiding difficulties.

In younger asymptomatic women, we also collected biopsy samples of ectocervix and endometrium from hysterectomy specimens. These samples were utilized to determine p27 expression in other tissues, for comparison with expression in the skeletal muscle (Fig. 9). In addition, we utilized our collection of frozen human corpora lutea (CL), from which some observations have been reported previously [21-23], in order to determine a relationship of p27 expression with some other cell cycle related proteins under specific circumstances (Fig. 10). All parts of the study were approved by the Institutional Review Board at our University of Tennessee Graduate School of Medicine. All women underwent the informed consent process and signed informed consent forms before participation.

\section{Immunohistochemistry}

Biopsy samples were mounted in $10 \times 10 \times 5 \mathrm{~mm}$ vinyl specimen molds (Tissue-Tek Cryomold Biopsy, Miles Inc. Diagnostic Division, Elkhart, IN) and embedded in O.C.T. compound (Miles). The molds with specimens were frozen in liquid nitrogen and stored at $-80^{\circ} \mathrm{C}$ until use. Frozen tissues were sliced into $7 \mu \mathrm{m}$ serial sections using a cryostat microtome with specimen retraction during return travel (Carl Zeiss Microm HM 505 E; MICROM Laborgeräte $\mathrm{GmbH}$, Waldorf, Germany). The slides were dried overnight at room temperature and stored at $4^{\circ} \mathrm{C}$. The immunohistochemical and histo- 


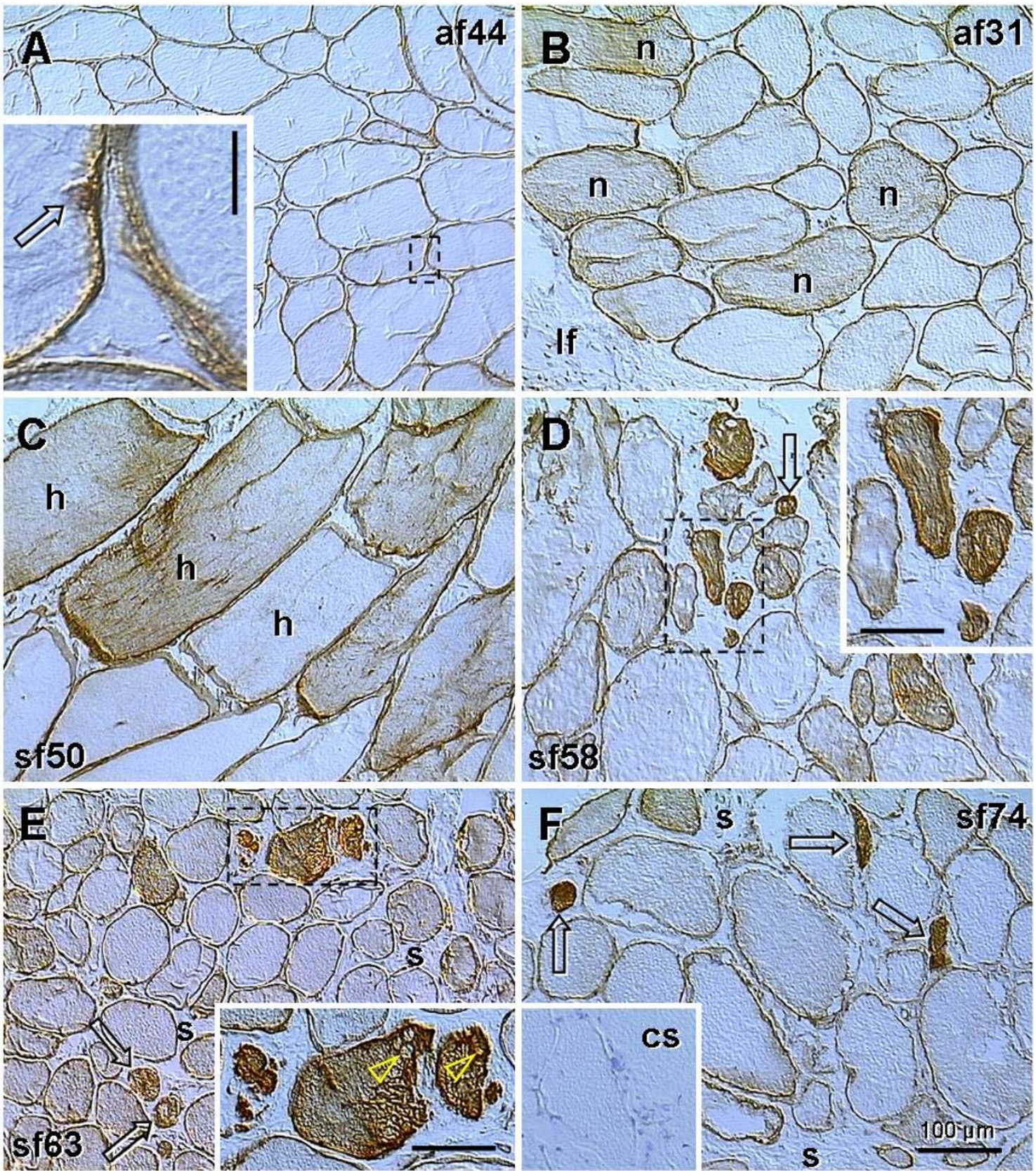

\section{Figure 3}

p27 expression (brown color) in asymptomatic (af) and symptomatic females (sf) at the ages indicated. Dashed boxes indicate areas shown in detail. A) Muscle fibers distant from the levator ani fascia show no cytoplasmic p27 expression. Inset shows strong staining of plasma membrane and adjacent nucleus (arrow). B) Some fibers adjacent to the levator fascia (If) often show weak cytoplasmic staining, but normal cell size (n). C) Hypertrophy of muscle fibers (h) is accompanied by variable p27 cytoplasmic expression (from none to moderate). D) Variable size of muscle fibers. Strong cytoplasmic staining accompanies regression of myotubes (arrow and dashed box). E) Regression of muscle fibers (arrows) is associated with cytoplasmic vacuolization (arrowheads in inset) and expanded stromal component (s). F) Expanded muscular stroma (s) contains remnants of muscle fibers (arrows). Inset shows control staining (cs). Bars in insets: vertical $=10 \mu \mathrm{m}$, horizontal $=50 \mu \mathrm{m}$. 


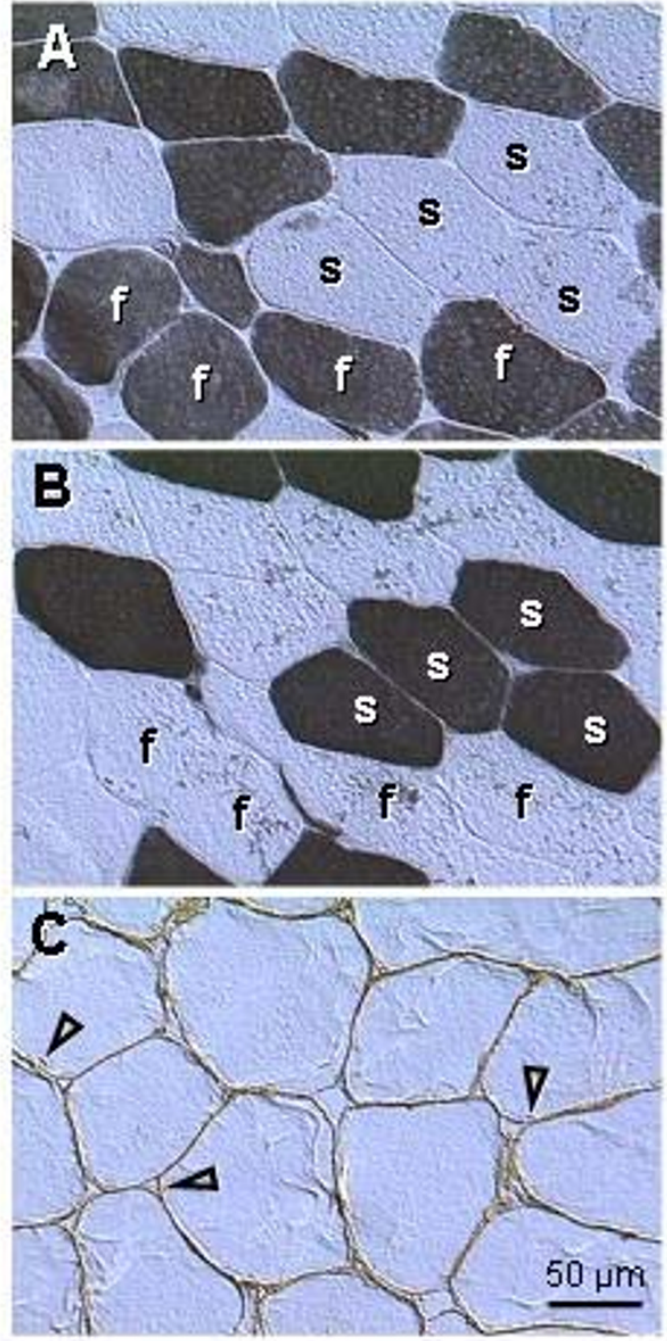

\section{Figure 4}

Type II fast- (A) and type I slow twitch fibers (B), and p27 expression (C) in levator ani muscle of an asymptomatic patient (age 38). A) Fast twitch fibers (f, dark color) alternate with slow twitch fibers (s). B) Parallel section to (A) shows staining of slow twitch fibers. C) Protein p27 is strongly expressed in plasma membrane and adjacent nuclei (arrowheads). Note a lack of cytoplasmic staining.

chemical procedures were performed within two weeks. If required, additional sections were obtained from frozen blocks.

The labeled streptavidin-biotin method was employed for peroxidase immunohistochemistry. All steps were performed at room temperature. Universal DAKO $\mathrm{LSAB}^{\circledR}{ }_{2}$ Peroxidase Kit (DAKO Corporation, Carpinteria, CA) was used according to the instructions provided by vendor with some modifications. Briefly, cryostat sections were fixed with acetone, dried for 30 minutes, and rinsed in phosphate buffered saline (PBS). Phosphate buffered saline was freshly prepared before staining from frozen $10 \times$ stock solution, and $\mathrm{pH}$ adjusted to 7.22. Mouse primary antibody, negative control reagent (DAKO), or PBS were applied for 15 minutes. Slides were washed twice in PBS, and link solution (biotinated antimouse antibody) was applied for 15 minutes. Subsequently, washed slides were incubated with streptavidinperoxidase conjugate for 15 minutes, washed again, and incubated 5 minutes in substrate-chromogen solution, rinsed with PBS, washed in distilled water, counterstained 2 minutes with Harris hematoxylin diluted 1:20, dehydrated, and mounted.

Purified mouse monoclonal antibody to p27 cell cycle inhibitor protein, clone G173-524, was purchased from PharMingen, San Diego, CA, and used at dilution 1:50. This antibody shows a single band in western blots (Fig. 2). Other primary antibodies used in some instances (Fig. 10) were mouse monoclonal antibodies to cyclin D1 (cD1, clone DCS-6, diluted 1:20, NeoMarkers, Fremont, CA), cyclin E (cE, HE111, 1:40, Santa Cruz Biotechnology, Santa Cruz, CA), Ki67 (Ki-67, 1:50, Dako Corporation, Carpinteria, CA), p21 Cip1/WAF1 (p21, clone 70, 1:25, Transduction Laboratories, San Diego, CA), and retinoblastoma protein (pRb, o5-218, 1:50, Upstate Biotechnology, Lake Placid, NY).

\section{Histochemistry}

To determine a character of muscle fibers (type II or type I) exhibiting changes in p27 expression, adjacent cryostat sections were stained for actomyosin adenosinetriphosphatase (ATPase). The procedure for the histochemical demonstration of actomyosin ATPase was employed as described by Goth and Samara [24]. Briefly, fixed sections were washed in rinse solution $[18 \mathrm{mM}$ calcium chloride in $100 \mathrm{mM}$ tris (hydroxymethyl) aminomethane (Tris), $\mathrm{pH}$ 7.8] for 1 minute, preincubated either in $50 \mathrm{mM}$ potassium acetate in $18 \mathrm{mM}$ calcium chloride at $\mathrm{pH} 4.35$ (for acid-stable myosin ATPase, type I slow twitch muscle fibers), or in $18 \mathrm{mM}$ calcium chloride in $100 \mathrm{mM}$ 2-amino-2-methyl-1-propanol at $\mathrm{pH}$ 10.4 (for alkali-stable myosin ATPase, type II fast twitch fibers). All sections were washed in rinse solution, then incubated at $37^{\circ} \mathrm{C}$ in solution containing $2.7 \mathrm{mM}$ ATP, 50 $\mathrm{mM}$ potassium chloride and $18 \mathrm{mM}$ calcium chloride in $100 \mathrm{mM}$ 2-amino-2-methyl-1-propanol at $\mathrm{pH} 9.4$ for 30 minutes, and washed in $1 \%$ calcium chloride. The sections were placed in $2 \%$ cobalt chloride solution for 3 minutes, washed in four 30-sec changes in $100 \mathrm{mM} 2-$ amino-2-methyl-1-propanol at $\mathrm{pH}$ 9.4, developed in 1\% ammonium sulfide solution for 3 minutes, washed in running tap water for 5 minutes, dehydrated in graded 

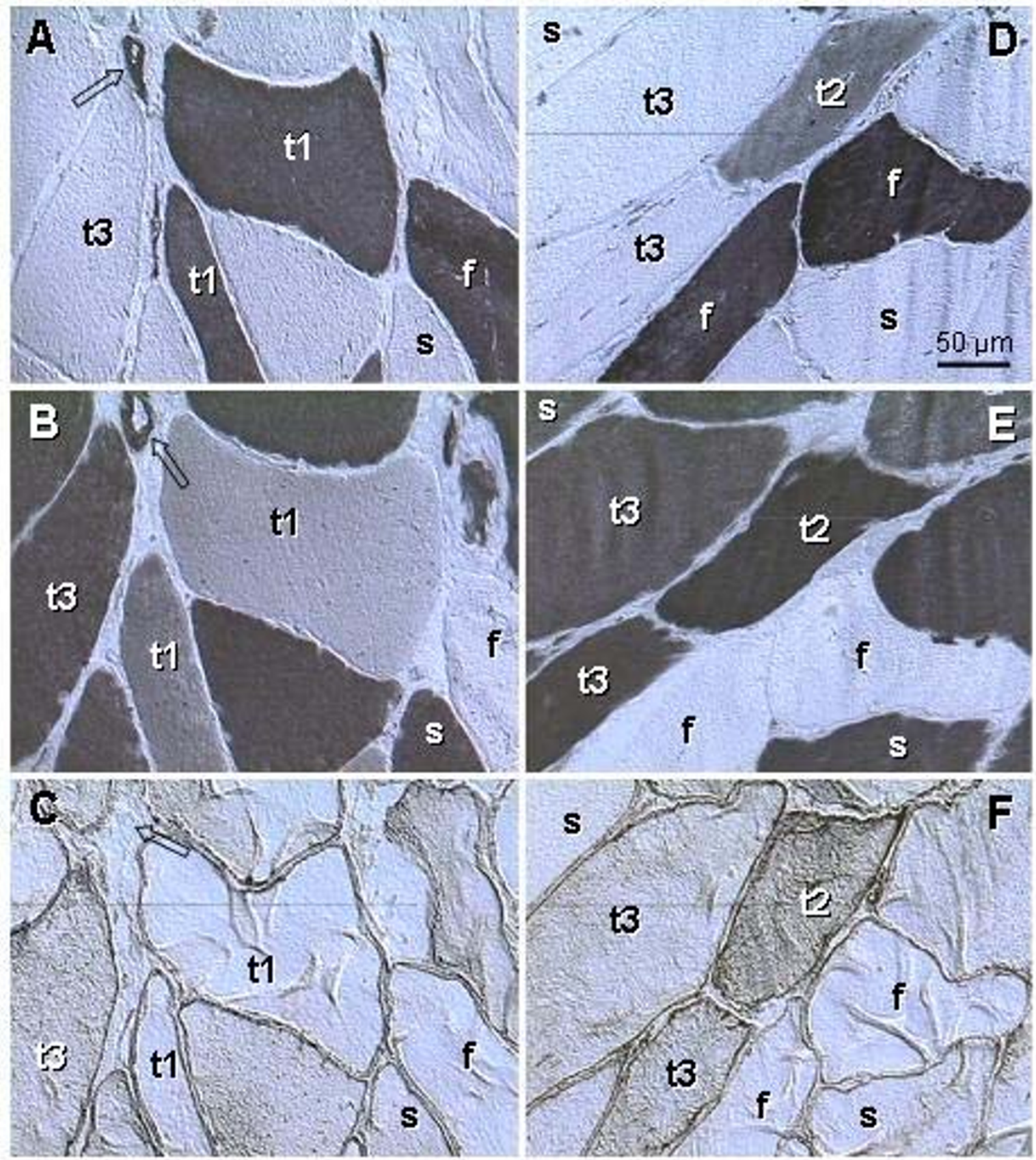

\section{Figure 5}

Type II (A, D) and type I staining (B, E) and p27 expression (C, F) in levator ani muscle of a symptomatic patient (age 50). A-C) Parallel sections. Fast twitch fibers (f) show type II staining only; slow twitch fibers (s) show type I staining only. Some fibers $(\mathrm{tl})$ show strong type II but also weak to moderate type I staining, without cytoplasmic p27 expression. Other fibers (t3) exhibit no type II, strong type I and moderate p27 staining. D-F) Parallel sections. Another type of fibers (t2) shows moderate type II and strong type I staining accompanied by moderate p27 expression, $\mathrm{tI}-\mathrm{t} 3$ indicates a presumptive course of the type II to type I transition. Arrows indicate vessels, which show both type II and type I staining and no p27 expression. 


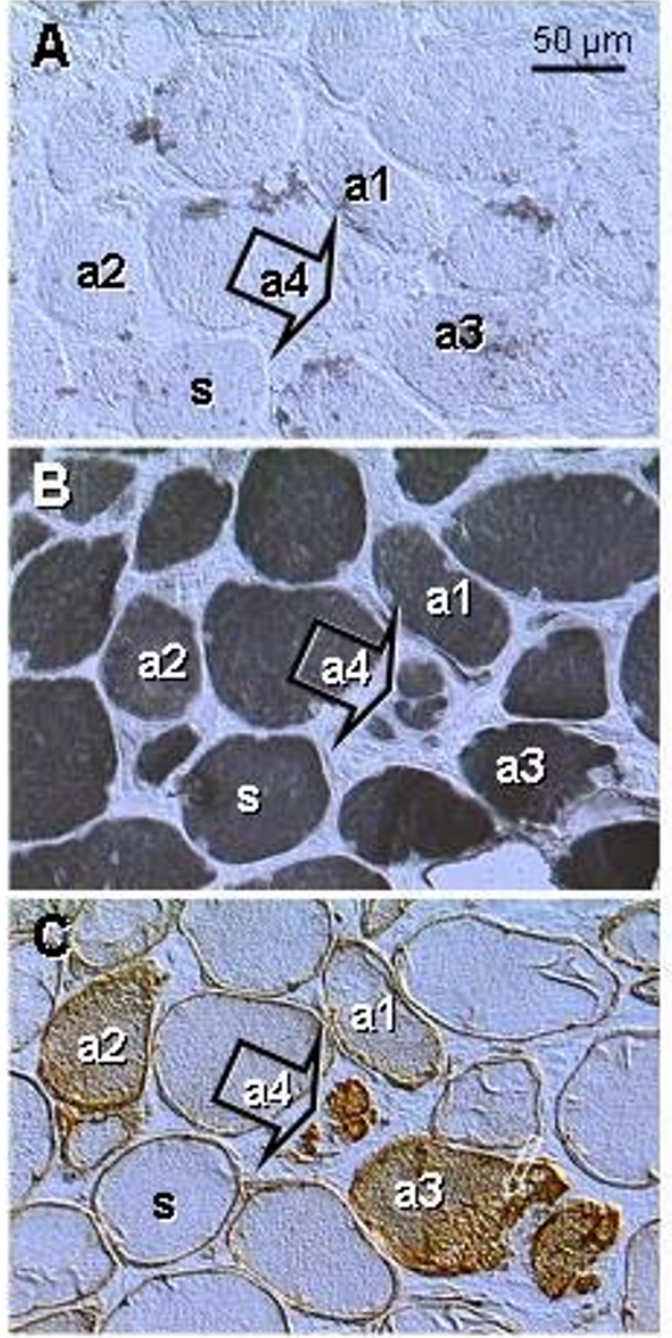

\section{Figure 6}

Type II (A) and type I staining (B) and p27 expression (C) in levator ani muscle of a symptomatic patient (age 63; see also Fig. 3E). Parallel sections. A) No fast twitch fibers are evident. B) All fibers show type I staining, including those exhibiting gradual diminution in size (a3 and a4). C) Cytoplasmic p27 expression gradually increases in regressing fibers (al to a4). al to a4 indicates presumptive course of apoptosis. s, genuine slow twitch fibers.

ethanol, cleared in xylene and mounted in Canada balsam. All chemicals were obtained from Sigma Chemical Co., St Louis, MO.

\section{Western blots}

For preparation of protein lysates from tissue samples (levator ani muscle; rectus fascia $=\sim$ negative control; and whole placenta $=$ low p27 positive control) 100 tissue sections ( $7 \mu \mathrm{m}$ thick) were collected into microcentrifuge tubes and lysed by adding ice-cold lysis buffer (20 mM Tris pH 7.5,200 mM NaCI, 0.25\% Nonidet P-40) containing $1 \mathrm{mM}$ sodium orthovanadate, $10 \mathrm{mM}$ sodium fluoride, and $1 \mathrm{mM}$ phenylmethylsulfonyl fluoride (400 $\mu \mathrm{l} /$ $100 \mathrm{mg}$ of tissue sections). After $15 \mathrm{~min}$ on ice, the lysates were sonicated by Sonicator ${ }^{\mathrm{TM}}$ Cell Disruptor (Heat Systems-Ultrasonic, Inc., Plainview, NY) for 5 seconds, and centrifuged at $11000 \times g$ for $10 \mathrm{~min}$ at $4^{\circ} \mathrm{C}$. Supernatants were stored at $-80^{\circ} \mathrm{C}$. We also prepared cell lysates from primary trophoblast cultures ( $=$ high p27 positive control), which were established and processed as described previously [18].

Protein concentrations were determined by Bradford assay (Bio-Rad, Hercules, CA). Equal amounts of protein (21 $\mu \mathrm{g}$; without boiling) were loaded onto reducing 10\% SDS-Tris-glycine polyacrylamide gels and transferred to nitrocellulose (Bio-Rad). For this study, the first five lanes were loaded with following lysates: Lane $1=$ whole placenta (WP, Fig. 2), lane $2=$ trophoblast cultured for 120 hours ( $\mathrm{TrC}$ ) and derived from the placenta loaded in lane 1 , lane 3 = rectus fascia (RF), lane $4=$ levator muscle from symptomatic female, age 63 (sLM), and lane $5=$ levator muscle from asymptomatic female (aLM). Remaining 5 lanes were loaded with molecular weight markers, and sLM, aLM, TrC and WP lysates. After transfer, the membrane was cut in two pieces, just in the front of the molecular weight markers. Membranes were washed in Tris-buffered saline containing 0.05\% Tween 20 (TBST) and non-specific binding sites were blocked by immersing the membrane in blocking reagent (0.5\% casein in TBST) for 1 hour at room temperature on an orbital shaker. After blocking, the membranes were briefly rinsed in two changes of TBST, and washed once for 15 minutes and twice for 5 minutes in fresh changes of TBST. Membrane with first five lanes was stained for p27 $(0.1 \mu \mathrm{g} / \mathrm{ml}$ in blocking reagent, clone G173-524, PharMingen) overnight at $4^{\circ} \mathrm{C}$ on an orbital shaker, followed by secondary antibody (see below). After development and film exposure, the membrane was washed and stained for actin $\left(0.25 \mu \mathrm{g} / \mathrm{ml}\right.$ in blocking reagent, clone $\mathrm{C}_{4}$, Boehringer Mannheim Corp., Indianapolis, IN). Remaining piece of membrane was processed with control staining (blocking reagent overnight at $4^{\circ} \mathrm{C}$ on an orbital shaker, followed by secondary antibody). After overnight incubation, membranes were washed and incubated with peroxidase labeled secondary antibody (goat antimouse IgG and IgM, pre-absorbed with human serum and diluted 1: 2000; Jackson Immunoresearch, West Grove, PA) for 1 hour at room temperature. The membranes were washed $1 \times 15 \mathrm{~min}, 2 \times 10 \mathrm{~min}$ and $4 \times 5 \mathrm{~min}$ in fresh changes of TBST, and incubated for 1 hour in blocking buffer at room temperature. Bound antibodies were detected by a chemiluminescent detection system 


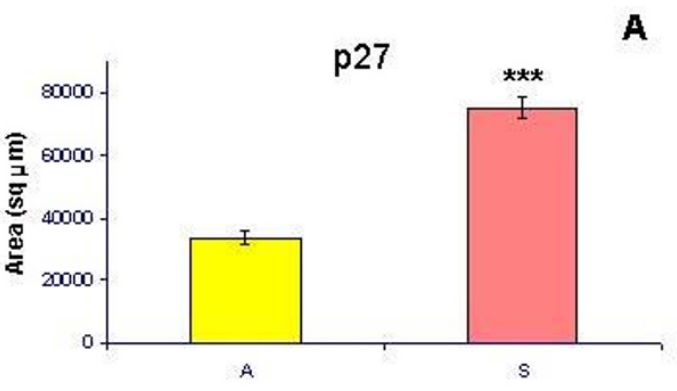

B

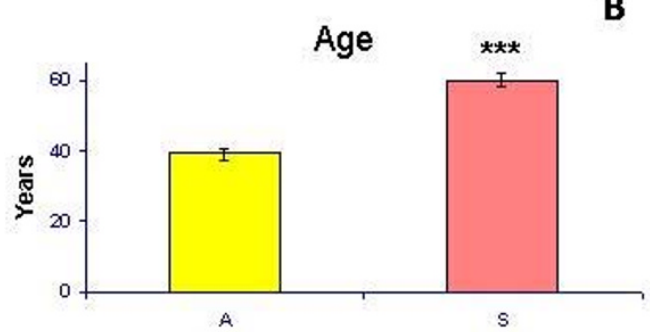

Figure 7

Quantitative evaluation of p27 expression (materials and methods) and comparison of the mean age in asymptomatic (A) and symptomatic patients (S). ${ }^{* * *}, P<0.000 \mathrm{I}$.

(ECL; Amersham Pharmacia Biotech, Piscataway, NJ) as recommended by the manufacturer's protocol . For exposure (first $5 \mathrm{~min}$, and second up to $2 \mathrm{~h}$ ) we used Kodak XAR film (Eastman Kodak, Rochester, NY).

\section{Image analysis}

Evaluation was performed on a Leitz DM RB (Leica Inc., Wetzlar, Germany) microscope equipped with differential interference contrast and a DEI-470 CCD Video Camera System (Optronics Engineering, Goleta, CA) with detail enhancement. The video images were captured by CG-7 color frame grabber (Scion Corporation, Frederick, MD) supported by Scion Image public software developed at the National Institutes of Health (Wayne Rasband, NIH, Bethesda, MD), and ported to Windows 98. The pictures were copied into the Microsoft $^{\circledR}$ PowerPoint ${ }^{\circledR} 97$ SR-2 (Microsoft Corporation, Redmont, WA) software, labeled, and saved in Portable Network Graphics format.

The immunohistochemical staining was quantified as an area in square micrometers (maximum area for whole screen was 307,200 sq micrometers at $100 \times$ magnification) occupied by the densities of peroxidase staining above the sample background, as described previously $[25,26]$. Briefly, the illumination of empty field (image background) was first adjusted to 40 in o to 255 scale. Measurement of an area of peroxidase staining was performed using the density slice method. The upper limit of the density slice was set at the 255 (maximum density value) and the lower limit at 150, sufficiently high above the hematoxylin staining of the nuclei, which does not exceed a density value 120 . Under these conditions, the measured area represented a crude value in square micrometers (e.g., 50,000). To obtain net values, each crude value was subtracted with the value of tissue background obtained from the same sample stained with a control staining (e.g., $500 \mu \mathrm{m}^{2}$, net value $=49,500 \mu \mathrm{m}^{2}$ ). Six measurements were performed on each biopsy sample. These six values represented a dataset for a single biopsy (patient) stained for a particular marker. Data in Fig. 7 represent means of 54 measurements from asymptomatic patients $(\mathrm{A} ; n=9)$ and 132 measurements from symptomatic patients $(S ; n=22)$.

To evaluate the density of p27 staining in western blots, we used a different approach. Scanned blot was transferred into the Scion Image, enlarged $4 \times$ (two times $2 \times$ ) and each lane was processed as follows. Small square area $(20 \times 20$ pixels) was used to obtain six background measurements above the p27 band. Subsequently, six measurements of staining within the p27 band (crude values) were obtained. Each crude value was subtracted with corresponding background value, giving the six net values of p27 density. The net values were used for statistical calculations.

\section{Statistical analysis}

Statistical analysis of data was performed using the Statistical Package of the Microsoft Excel 97 SR-2 (Microsoft Corporation, Redmont, WA) professional version (Descriptive Statistics), and GraphPad InStat (GraphPad Software, Inc., San Diego, CA, [www.graphpad.com] ) software. The Mann-Whitney Test was used to compare two columns. Repeated Measures Analysis of Variance, followed by the posthoc Student-Newman-Keuls Multiple Comparison test, was used for a comparison of multiple columns. Values of $P<0.05$ were considered significantly different. Power calculations were performed using GraphPad StatMate version 1.o1i (GraphPad Software). A threshold $P$ value (alpha) of 0.05, two tailed, was selected.

\section{Results and Discussion Western blot analysis}

Fig. 2A shows that the antibody to p27, clone G173-524, produces a single band at $27 \mathrm{kDa}$ in all investigated tissues, and no nonspecific staining is apparent with secondary antibody alone. This indicates that all 


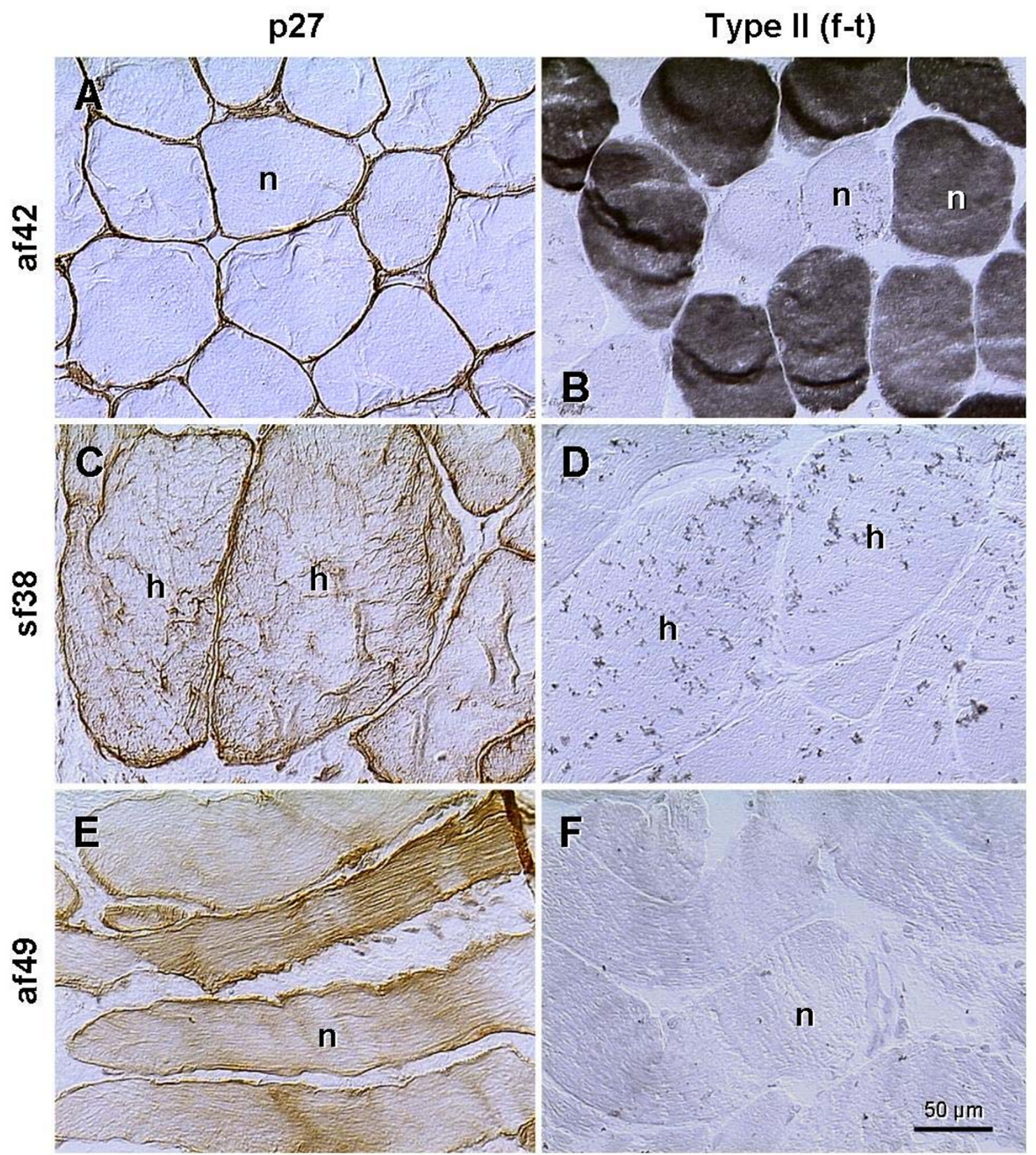

Figure 8

Staining for p27 and type II fast twitch fibers (f-t) in the levator ani of an asymptomatic female, age 42 (af42), youngest symptomatic female (sf38) and the oldest asymptomatic female (af49) from our collection of cases, $n$, normal size fibers; $h$, hypertrophy. Details in text. 


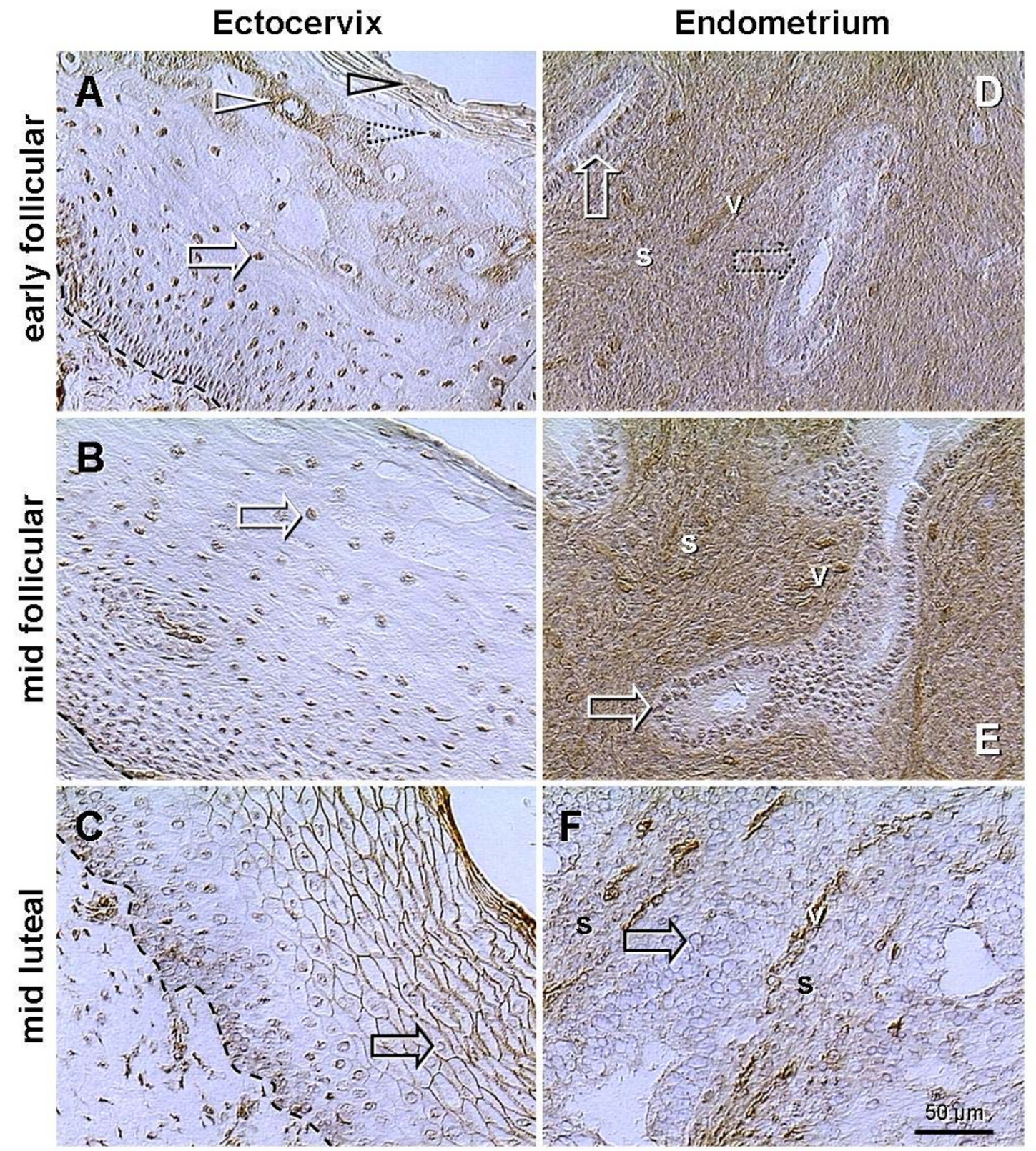

\section{Figure 9}

Changes of p27 expression in the epithelium of ectocervix and in endometrium during the menstrual cycle (rows show early and mid follicular phases and mid luteal phase). A) In addition to nuclear staining (white arrow), the cytoplasmic p27 expression is apparent in the upper intermediate layers (white arrowhead) and cytoplasmic and plasma membrane staining in the surface cells (black arrowhead). These two layers are divided by cells showing nuclear p27 only (dotted arrowhead). B) Cells show only nuclear p27 throughout the epithelium. C) Strong surface expression in mature and aging epithelial cells. Note that within cells, the traces of p27 staining are confined to the nuclear envelope (black arrow). D) Strong staining of the stroma and patchy nuclear p27 expression in some (developing) glands (dotted arrow). E) Increased stromal and nuclear staining (vs. D). F) p27 is severely depleted from the nuclei of glandular cells (black arrow) and stroma, but persists in vessels. Dashed line, basement membrane; s, endometrial stroma; $v$, vessels. 

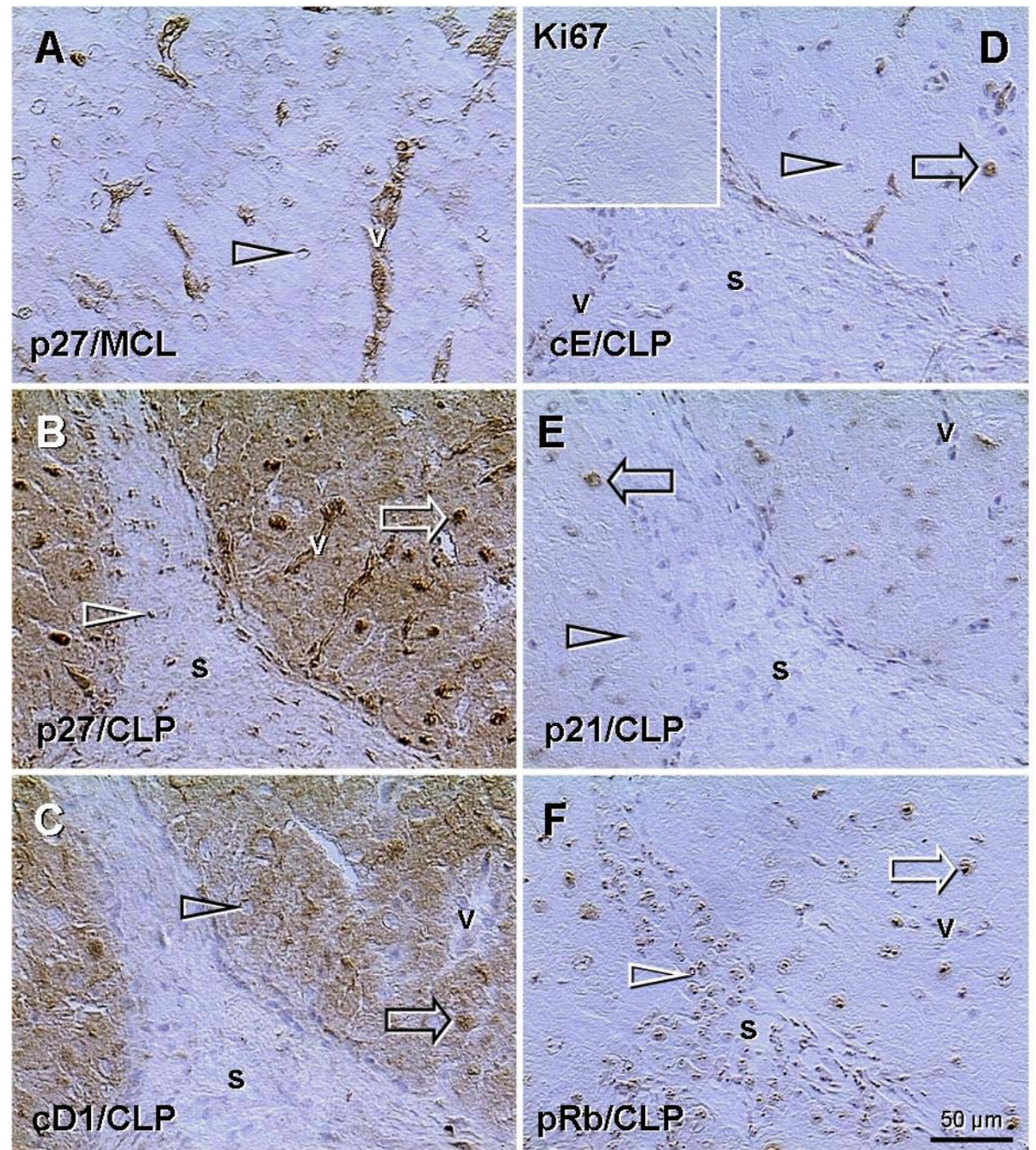

Figure 10

Indirect peroxidase immunohistochemistry of mature corpus luteum of the cycle (MCL, panel $A)$ and $C L$ of pregnancy (CLP, panels B-F) with monoclonal antibodies to p27, cyclin DI, cyclin E, Ki67, p2I, and pRb, as indicated in panels. A) Arrowhead indicates traces of p27 (nuclear envelope) in luteal cells of the mature CL of the cycle; note strong expression in luteal vessels. B) Preservation of the CL during pregnancy is associated with very strong nuclear and moderate cytoplasmic expression of p27 in luteal cells, in addition to the vessels. C) Similar cytoplasmic staining of luteal cells is apparent for cyclin DI (note unstained vessels), but not for other cell cycle proteins investigated (panels D-F). Note that all nuclei of luteal (white arrows) and stromal cells (white arrowheads) are stained in panels B and F, but only some nuclei of luteal cells are stained in panels C-E (black arrows) and other are unstained (black arrowheads), including stroma. Insert in (D) indicates a lack of Ki67 dividing cells in the CL of pregnancy (and no background staining); v, luteal vessels; s, stroma. 
immunohistochemical observations should be ascribed to the specific expression of p27 protein. In western blot, the most prominent p27 expression was in the late trophoblast culture $(120 \mathrm{~h})$, as expected from our previous study [18]. When compared to trophoblast culture, the p27 expression in levator ani muscles was lower, but higher then weak expression in whole placenta and rectus fascia.

Fig. 2B shows quantitative evaluation (materials and methods) of the blot in panel A. The statistical evaluation showed no significant difference between two cases included (symptomatic and asymptomatic female) in density of p27 expression in levator ani muscles.

\section{Immunohistochemistry}

Expression of p27 in levator ani muscle fibers of control asymptomatic women was restricted to the plasma membrane and adjacent nuclei (Fig. 3A, and inset). Some muscle fibers close to the levator fascia often showed weak cytoplasmic staining but no change in the cell size (Fig. 3B). Pre- and perimenopausal symptomatic patients showed hypertrophy of muscle fibers, and this was associated with variable cytoplasmic p27 expression, ranging from none to moderate (Fig. $3 \mathrm{C}$ ). Biopsy samples obtained from symptomatic women in advanced postmenopause showed diminution of size in some fibers accompanied by strong cytoplasmic p27 expression (Fig. 3D, and inset). This was accompanied by appearance of cytoplasmic vacuoles in regressing muscle fibers (Fig. $3 \mathrm{E}$, and inset) and by an increase in the stromal component (s. Fig. $3 \mathrm{E}$ and $3 \mathrm{~F}$ ). Control procedure showed no non-specific staining (inset. Fig. 3F).

These data indicate that hypertrophy of muscle fibers may be accompanied by moderate p27 cytoplasmic expression. Further increase in cytoplasmic staining (p27 overexpression) accompanies shrinking and fragmentation of muscle fibers. This was characteristic for symptomatic women in advanced postmenopause, suggesting that hormonal changes accompanying postmenopause, such as diminution of ovarian sex steroid production, might contribute to the degenerative changes in the levator ani muscle.

From the ovarian sex steroids, the role of diminishing estrogen levels is questionable, since the levator ani muscle fibers do not express estrogen receptor alpha $[2,26]$. It is not known if estrogen receptor beta is expressed, but levator ani muscle fibers show nuclear expression of progesterone and androgen receptors [26]. Altered ovarian function in the premenopause can be observed as early as age 43 years, and includes hyperestrogenism and hypergonadotropism, accompanied by decreased progesterone secretion [27]. During the transition from pre- to postmenopause both estrogen and androgen levels also decline [28]. These hormonal alterations may well be responsible for the increased gynecological morbidity that characterizes this period of life, including structural and molecular changes in levator ani muscle and increasing incidence of pelvic floor disorders.

\section{Diminution of fast twitch fibers}

We also studied histochemical staining (type II and type I fibers) along with protein p27 expression. Fig. 4, A,B,C, is a biopsy from an asymptomatic women (age 38 ) exhibiting a proportional number of fast- (f; Fig. 4A) and slow twitch fibers (s; Fig. 4B), and strong surface and nuclear but no cytoplasmic p27 expression (Fig. 4C).

Fig. 5 is from a symptomatic female (age 50). Some muscle fibers exhibited both type I and type II staining, representing the "transitory" (t1 and t2) types. This was accompanied by p27 cytoplasmic expression in t2 fibers. In addition, some fibers showed type I staining accompanied by p27 cytoplasmic expression ( $\mathrm{t} 3$ fibers). This contrasted with a quiescent (genuine) fast- (f) and slow twitch (s) fibers, which showed either type I or type II staining and no p27 cytoplasmic expression. The "transitory" fibers often exhibited hypertrophy.

Occurrence of both type II and type I staining in the same striated muscle fiber has not been described. When using the term "transitory" fibers, we are aware that a static picture can not explain the dynamic process. However, the "transitory" fibers show both type II and type I staining, cytoplasmic p27 expression suggestive of ongoing differentiation $[17,18]$, and marked hypertrophy. The occurrence of "transitory" fibers was also associated with a decline in the number of fibers exhibiting the type II staining only, which suggests a type II into type I transition. Also, a lack of muscle cell shrinking and fragmentation during this process indicates that type II fibers do not degenerate but are transformed into the type I fibers.

\section{Shrinking and fragmentation of muscle fibers}

Fig. 6A shows a lack of type II fibers in postmenopausal patient (age 63). Type I staining was characteristic for all fibers present, including those exhibiting a diminution in size (Fig. 6B). Staining for p27 revealed appearance of weak (a1) to moderate cytoplasmic expression (a2) in some otherwise normal fibers. Very strong p27 expression was characteristic for the fibers exhibiting cytoplasmic vacuolization (a3, see also inset, Fig. $3 \mathrm{E}$ ) and fragmentation (a4, arrow), i.e., morphologic features characteristic for the cells undergoing apoptotic cell death [29]. 


\section{Quantitative evaluation of p27 expression}

Fig. 7A shows cumulative data on p27 expression in levator ani muscle from asymptomatic and symptomatic patients. The mean p27 expression in symptomatic patients (age range $38-73$ years) was much higher $(P<0.0001)$ when compared to controls (age range $31-49$ years). The power for number of cases was $80 \%$, for number of measurements 99\%. However, there was also a highly significant difference in mean age between the groups (Fig. 7B; P < 0.0001, power 99\%). At present, we are not able to determine if in asymptomatic postmenopausal women the p27 expression in levator ani muscles also increases with age, since non-urogynecological surgery, except cancer (excluded), is exceptional in such cases.

\section{p27 cytoplasmic expression and muscle abnormality}

An important question is whether the increase in cytoplasmic p27 expression correlates with the age of the patients and the severity of the muscle abnormality. Fig. 8 shows a lack of cytoplasmic p27 staining of levator ani muscle in another control female (age 42, panel A) exhibiting normal size of muscle fibers (n) and high proportion of type II fibers (panel B). Panels C and D show a situation in the youngest patient from symptomatic group (age 38; pelvic organ prolapse). The muscle fibers show marked hypertrophy (h) and cytoplasmic p27 expression (panel C) associated with a lack of type II staining (panel D). On the other hand, panels $\mathrm{E}$ and $\mathrm{F}$ show a situation in the oldest female in asymptomatic group. The size of fibers is normal, but moderate p27 cytoplasmic staining is evident (panel E). This is, however, accompanied by a lack of fast twitch fibers (panel F).

Hence, even in the presence of moderate cytoplasmic p27 expression in muscle fibers of normal size (Fig. 8E), which accompanies depletion of fast twitch fibers (panel
F), the woman may remain continent, without an actual evidence of pelvic floor disorder. On the other hand, cytoplasmic p27 expression accompanying hypertrophy of muscle cells (panel C) was accompanied by PFD. These observations indicate that an increase in cytoplasmic p27 staining may accompany normal female aging (age 49), along with the diminution of fast twitch fibers in otherwise normal muscles, and without manifestation of PFD. Conversely, levator ani from younger symptomatic patient (age 38) exhibited hypertrophy of muscle cells, accompanied by increased cytoplasmic p27 expression and manifestation of PFD. Altogether, the cytoplasmic p27 expression is indicative of fast to slow twitch transition of levator ani myotubes, but not necessarily indicative of manifestation of PFD, assuming normal muscle morphology.

Table 1 summarizes our observations, and suggests possible pathways for the transition of type II to type I fibers and progression of degeneration of levator ani muscle cells. Note that a lack of type I staining is characteristic for fast twitch fibers only (row 1), and a lack of p27 cytoplasmic expression is characteristic for the "resting" fastand slow twitch fibers and fibers in "early transition" (rows 1, 2 and 5). Only fibers in row 3 show type II, type I and p27 cytoplasmic staining, and a lack of type II staining is apparent from row 4 on. During transition, the fast twitch fibers may increase in size, and gradually change into the slow twitch fibers exhibiting regular size. Fibers in "advanced transition" (rows 3 and 4), and those undergoing degeneration (rows 6 and 7), are associated with cytoplasmic p27 expression. Although rows 4 and 6 are similar, the latter is associated with the regression of many accompanying myotubes (see Fig. 3, D-F, and Fig. 6).

Table I: Semiquantitative evaluation of the fast to slow twitch transition

\begin{tabular}{|c|c|c|c|c|c|}
\hline $\begin{array}{l}\text { Stage of muscle fiber } \\
\text { (myotube) differentiation }\end{array}$ & $\begin{array}{l}\text { Fiber } \\
\text { Type }^{\mathrm{a}}\end{array}$ & $\begin{array}{l}\text { Type II } \\
\text { stainingb }\end{array}$ & $\begin{array}{l}\text { Type I } \\
\text { staining }\end{array}$ & $\begin{array}{l}\text { Cytoplasmic } \\
\text { p27 }\end{array}$ & Fiber size \\
\hline I - resting fast (young) & fast & +++ & - & - & normal \\
\hline 2 - transitory I & trans I & +++ & ++ & - & normal or increased \\
\hline 3 - transitory 2 & trans 2 & ++ & +++ & + & normal or increased \\
\hline 4 - transitory 3 & trans 3 & - & +++ & ++ & normal or increased \\
\hline 5 - resting slow (mature) & slow & - & +++ & - & normal \\
\hline 6 - early deg (aging) & $\operatorname{deg} 1-2$ & - & +++ & ++ & normal \\
\hline 7 - late deg (regressing) & $\operatorname{deg} 3-4$ & - & +++ & +++ & fragmented \\
\hline
\end{tabular}

\footnotetext{
a fast $=$ fast twitch fibers; trans $I=$ early, trans 2 = mid, trans $3=$ late transition of fast- to slow twitch fibers; slow = slow twitch fibers; deg $1-2=$ early, deg 3-4 = advanced degeneration (shrinking and fragmentation; see Fig. 3, D-F for examples, and Fig. 6 for staging). ${ }^{b}$ Semiquantitative grading: -, none; +, weak; ++, moderate; +++, strong (see Fig. 3 to 6 for corresponding densities).
} 


\section{How unique is the unusual p27 expression for the skeletal muscle?}

p27 is a cyclin-dependent kinase (cdk) inhibitor, and is normally expected to be localized to the cell nucleus. In normal cells, p27 is sequestered and its activity gradually decreases as cells reach $\mathrm{S}$ phase, p27 preferentially binds cyclin D-cdk4, but lower levels of cdk4 (in TGF- $\beta$ treated cells) allow p27 to be available for binding to the cyclin Ecdk2 and cyclin A-cdk2. p27 is structurally related to the p21, having a similar sequence in the $\mathrm{N}$-terminal region. Inhibition of cell cycle by p27 and p21 is complemented by retinoblastoma protein $\mathrm{pRb}$, and other cell cycle inhibitors (tumor suppressor proteins) [30-32]. In reality, besides their role in the regulation of the cell cycle, the cdk inhibitors, in association with D-type cyclins, cyclin $\mathrm{E}$, and cdk2, play a direct role in the stimulation of epithelial cell differentiation [33]. p27 is also transiently expressed in developing myotomes and enhances myogenesis [34].

The localization of p27 to plasma membrane and the cytoplasm in normal and abnormal striated muscle in vivo raises a question how unique these unusual expressions are for the skeletal muscle cells. The observations, summarized in Table 1, indicate that cytoplasmic expression of p27 is related to two dynamic processes, (i) advanced "transition" of fast to slow twitch fibers (rows 3 and 4, Table 1), and (ii) gradual degeneration of muscle cells (rows 6 and 7). On the other hand, regardless of the status (quiescent fast and slow twitch fibers, myotubes in transition or degenerating) the muscle cells exhibit plasma membrane p27 staining.

Excellent models for the studies of cell differentiation in vivo are stratified squamous epithelia [35], particularly those that are easily to collect, such as the samples of epithelium of ectocervix from hysterectomy specimens. In addition, the epithelium of ectocervix is hormonally dependent, and shows certain changes along with the fluctuations of levels of ovarian steroids during the menstrual cycle. Another, even more hormonally dependent tissue is endometrium. Fig. 9 shows p27 expression in the ectocervix and corresponding endometrium (from the same uteri).

In ectocervix, during the early follicular phase, when increasing levels of estrogens stimulate proliferation of stem cells and subsequent shift of cells between the layers - toward surface, some mature epithelial cells (upper intermediate layers) showed cytoplasmic p27 staining (white arrowhead, Fig. 9A). p27 expression was also apparent in the surface (degenerating) cells (black arrowhead) but not between these two layers (dotted arrowhead). This resembles the transition of type II into type I fibers, and degeneration of myotubes. In the mid follicular phase, p27 staining was restricted to the nuclei (white arrow, panel B), but during the luteal phase, when the influence of estrogens is accompanied by progesterone, the nuclear p27 diminished (black arrow, panel C), and mature and aged epithelial cells showed surface p27 expression, similar to that in all types of muscle fibers.

In endometrium, the early follicular phase was associated with strong stromal p27 expression (s, panel D), accompanied by variable nuclear expression in epithelium of narrow glands (arrows). In the mid follicular phase, stromal and nuclear p27 staining increased further (panel E), but luteal phase endometrium showed diminution of both (panel F). Altogether, these observations indicate that, in addition to the striated muscle cells, the cytoplasmic and plasma membrane p27 expression can be observed (under certain circumstances) in other cell types. Yet, the cytoplasmic p27 expression in abnormal skeletal muscles also appears to be temporary, i.e., during the transition (type II to type I) and terminal regression of myotubes.

Moreover, a combined action of estrogens and progesterone, characteristic for the luteal phase, appears to cause a diminution of nuclear p27 expression in the epithelium of ectocervix and endometrium (Fig. 9, C and 9F). Curiously, the remnants of p27 staining were confined to the nuclear envelope (arrows, Fig. 9, C and 9F; see also Fig. 10A), suggesting a degradation of p27 in the nuclear core, and/or a defect of p27 synthesis/transport. These tissues, however, do posses nuclear receptors for both steroids $[36,37]$.

We did not detect similar fluctuation of nuclear p27 expression in muscle fibers, yet myotubes do not exhibit estrogen receptor expression [2,26]. However, estrogen, progesterone and androgen receptors are expressed in the principal cells (fibroblasts) of levator ani fascia [26], which represents another important component of the pelvic floor involved in its suspensory function, and might be a target of the complex effect of hormonal fluctuations during the cycle, and influenced by the hormone replacement therapy in postmenopausal women.

Some recent studies indicate that long-term continuous estrogen replacement is not beneficial, yet may worsen or induce the manifestation of PFD [38,39], possibly through the suppression of sex steroid receptor expression in the pelvic floor. Since progesterone and androgen receptors are also expressed in the levator ani muscle, and long-term estrogen replacement therapy appears to suppress their expression [26], both the levator ani fascia and muscle might be affected by continuous estrogen replacement. 


\section{Correlation of cytoplasmic p27 with other cell cycle pro- teins}

Cytoplasmic p27 expression in the skeletal muscle and epithelium of ectocervix raises the question if it is associated with some other cell cycle proteins, which otherwise show characteristic nuclear localization. We investigated ovarian CL, another interesting structure for the studies of cell differentiation and degeneration in vivo [21-23], and found that cytoplasmic p27 expression is constantly present in the CL of pregnancy.

Fig. 10, panel A, shows that the mature CL of the menstrual cycle has p27 expression limited to the luteal vessels; luteal cells show only traces of nuclear p27 (arrow) and no cytoplasmic staining. However, in the CL of pregnancy, nuclei of luteal cells show abundant p27 expression (arrow, panel B), accompanied by moderate cytoplasmic staining. Nuclear p27 is also evident in stromal cells (s, arrowhead). When compared to p27, the cyclin D1 is expressed in some nuclei of luteal cells only (arrow, panel C) and absent from the stromal cells in the CL of pregnancy. However, the cytoplasmic expression of cyclin D1 is similar to that of p27. Staining for cyclin E (panel D) shows expression in occasional nuclei. The insert shows a lack of $\mathrm{Ki} 7^{+}$dividing cells. Expression of p21 (panel E) is similar to that of cyclin E. The pRb (panel F) shows nuclear expression similar to that of p27, including stromal cells, but no cytoplasmic staining of luteal cells.

These observations indicate that cytoplasmic expression of p27 correlates with that of cyclin D1. One may speculate that the cdk4 is involved, since p27 has a preferential affinity to the cyclin D-cdk4 complex. If the cytoplasmic p27 expression in abnormal muscle cells is accompanied by staining for cyclin D1, remains, however, to be established.

\section{Conclusions}

This is a first report on the p27 protein expression in normal and abnormal human striated muscle cells in vivo. Normal muscle fibers show strong nuclear and plasma membrane expression. Cytoplasmic p27 staining accompanies perimenopausal transition of type II to type I fibers. In the absence of other muscle abnormalities, the diminution of type II fibers alone is not necessarily accompanied by PFD. Pelvic floor disorders in perimenopausal patients are associated with the hypertrophy of muscle cells and moderate cytoplasmic p27 expression, while PFD during advanced postmenopause are associated with shrinking and fragmentation of muscle cells and cytoplasmic p27 overexpression. The cytoplasmic and plasma membrane p27 expression is not unique to the muscle cells, since it can also be detected to occur, at least temporarily, in other cell types.

\section{List of Abbreviations}

PFD, pelvic floor disorders; p27, p27 kip1; PBS, phosphate-buffered saline; CL, corpus luteum or corpora lutea; cdk, cyclin-dependent kinase; p21, p21 Cip1/WAF1.

\section{Competing interests}

\section{None declared.}

\section{Acknowledgments}

Authors thank the Editors for the selection of highly competent reviewers, and to the reviewers for their exceptionally professional and constructive criticism regarding both general significance of abnormal p27 expression in muscle fibers and clinical aspects of the manuscript. This work was supported in part by the Physicians' Medical Education and Research Foundation grant \#93040, Knoxville, Tennessee, to A.B.

\section{References}

I. Norton PA: Pelvic floor disorders: the role of fascia and ligaments. Clin Obstet Gynecol 1993, 36:26-938

2. Bernstein IT: The pelvic floor muscles: muscle thickness in healthy and urinary-incontinent women measured by perineal ultrasonography with reference to the effect of pelvic floor training. Estrogen receptor studies. Neurourol Urodyn 1997, 16:237-275

3. Gilpin SA, Gosling JA, Smith AR, Warrell DW: The pathogenesis of genitourinary prolapse and stress incontinence of urine. $A$ histological and histochemical study. Br J Obstet Gynaecol 1989, 96:15-23

4. Benson JT: Female Pelvic Floor Disorders, Investigation and Management. New York, WW Norton \& Company, 1992

5. Brubaker LT, Saclarides T]: The Female Pelvic Floor. Disorders of Function and Support. Philadelphia, FA Davis Company, 1996

6. Shafik A: Levator ani muscle: new physioanatomical aspects and role in the micturition mechanism. World J Urol 1999 , | 7:266-273

7. DeLancey JO, Starr RA: Histology of the connection between the vagina and levator ani muscles. Implications for urinary tract function. I Reprod Med 1990, 35:765-77|

8. DeLancey JO: The pathophysiology of stress urinary incontinence in women and its implications for surgical treatment. World J Urol 1997, I 5:268-274

9. Jozwik M: Stress urinary incontinence in women - an overuse syndrome. Med Hypotheses 1993, 40:381-382

10. Kjolhede P, Ryden G: Clinical and urodynamic characteristics of women with recurrent urinary incontinence after Burch colposuspension. Acta Obstet Gynecol Scand 1997, 76:46 I-467

II. Beersiek F, Parks AG, Swash M: Pathogenesis of ano-rectal incontinence. A histometric study of the anal sphincter musculature. I Neurol Sci 1979, 42: I I I- I 27

12. Failes D, Killingback M, Stuart M, De Luca C: The surgical treatment of anal incontinence. Aust N Z J Surg 1979, 49:345-349

13. Shafik A: Role of pudendal canal syndrome in the etiology of fecal incontinence in rectal prolapse. Digestion 1997, 58:489-493

14. Sandri M, Carraro U: Apoptosis of skeletal muscles during development and disease. Int J Biochem Cell Biol 1999, 3 I:I373-I390

15. Chu CY, Lim RW: Involvement of p27(kip I) and cyclin D3 in the regulation of cdk2 activity during skeletal muscle differentiation. Biochim Biophys Acta 2000, I 497:175-185

16. Polyak K, Lee MH, Erdjument-Bromage H, Koff A, Roberts JM, Tempst P, Massague J: Cloning of p27Kip I, a cyclin-dependent kinase inhibitor and a potential mediator of extracellular antimitogenic signals. Cell 1994, 78:59-66

17. Lloyd RV, Erickson LA, Jin L, Kulig E, Qian X, Cheville JC, Scheithauer BW: p27kip I: a multifunctional cyclin-dependent kinase inhibitor with prognostic significance in human cancers. Am J Pathol 1999, 154:313-323

18. McKenzie PP, Foster JS, House S, Bukovsky A, Caudle MR, Wimalasena J: Expression of $\mathbf{G I}$ cyclins and cyclin-dependent kinase-2 activity during terminal differentiation of cultured human trophoblast. Biol Reprod 1998, 58:1283-1289 
19. Katayose Y, Kim M, Rakkar AN, Li Z, Cowan KH, Seth P: Promoting apoptosis: a novel activity associated with the cyclin- dependent kinase inhibitor p27. Cancer Res 1997, 57:544 I-5445

20. Fujieda S, Inuzuka M, Tanaka N, Sunaga H, Fan GK, Ito T, Sugimoto C, Tsuzuki $\mathrm{H}$, Saito $\mathrm{H}$ : Expression of p27 is associated with Bax expression and spontaneous apoptosis in oral and oropharyngeal carcinoma. Int J Cancer I999, 84:3 I5-320

21. Bukovsky A, Caudle MR, Keenan JA, Wimalasena J, Upadhyaya NB, Van Meter SE: Is corpus luteum regression an immune-mediated event? Localization of immune system components, and luteinizing hormone receptor in human corpora lutea. Biol Reprod 1995, 53: I 373-1 384

22. Bukovsky A, Caudle MR, Keenan JA, Wimalasena J, Upadhyaya NB, Van Meter SE: Is irregular regression of corpora lutea in climacteric women caused by age-induced alterations in the "tissue control system"? Am J Reprod Immunol I996, 36:327-34 I

23. Bukovsky A, Caudle MR, Keenan JA, Elder RF: Immune system involvement in the regulation of ovarian function: an immunohistochemical study of the developing and adult human ovary and ovarian cancer. Microsc Res Tech 2001

24. Guth L, Samaha FJ: Procedure for the histochemical demonstration of actomyosin ATPase. Exp Neurol 1970, 28:365-367

25. Bukovsky A, Ayala ME, Dominguez R, Keenan JA, Wimalasena J, McKenzie PP, Caudle MR: Postnatal androgenization induces premature aging of rat ovaries. Steroids 2000, 65:190-205

26. Copas P, Bukovsky A, Asbury B, Elder RF, Caudle MR: Estrogen, progesterone and androgen receptor expression in levator ani muscle and fascia. J Womens Health Gend Based Med 200I

27. Santoro N, Brown JR, Adel T, Skurnick JH: Characterization of reproductive hormonal dynamics in the perimenopause. J Clin Endocrinol Metab 1996, 81:|495-I50|

28. Overlie I, Moen MH, Morkrid L, Skjaeraasen JS, Holte A: The endocrine transition around menopause - a five years prospective study with profiles of gonadotropines, estrogens, androgens and SHBG among healthy women. Acta Obstet Gynecol Scand 1999, 78:642-647

29. Stanulis BM-Praeger: Cellular senescence revisited: a review. Mech Ageing Dev 1987, 38: I-48

30. Yeargin J, Cheng J, Yu AL, Gjerset R, Bogart M, Haas M: P53 mutation in acute $\mathbf{T}$ cell lymphoblastic leukemia is of somatic origin and is stable during establishment of $T$ cell acute lymphoblastic leukemia cell lines. J Clin Invest 1993, 91:2 III2117

31. Toyoshima H, Hunter T: p27, a novel inhibitor of Gl cyclin-Cdk protein kinase activity, is related to $\mathrm{p} 2 \mathrm{I}$. Cell I994, 78:67-74

32. Loden M, Nielsen NH, Roos G, Emdin SO, Landberg G: Cyclin E dependent kinase activity in human breast cancer in relation to cyclin E, p27 and p2I expression and retinoblastoma protein phosphorylation. Oncogene 1999, 18:2557-2566

33. Tian JQ, Quaroni A: Involvement of p2I(WAFI/CipI) and p27(Kip I) in intestinal epithelial cell differentiation. Am J Physiol 1999, 276:CI245-CI258

34. Zabludoff SD, Csete M, Wagner R, Yu X, Wold BJ: p27Kip I is expressed transiently in developing myotomes and enhances myogenesis. Cell Growth Differ I998, 9:I-II

35. Bukovsky A, Caudle MR, Keenan JA, Upadhyaya NB, Van Meter S, Wimalasena J, Elder RF: Association of mesenchymal cells and immunoglobulins with differentiating epithelial cells. $B M C$ Dev Biol 200I, I:II

36. Blakeman PJ, Hilton P, Bulmer JN: Oestrogen and progesterone receptor expression in the female lower urinary tract, with reference to oestrogen status. $B J U$ Int 2000, 86:32-38

37. Habiba MA, Bell SC, Al Azzawi F: The effect of hormone replacement therapy on the immunoreactive concentrations in the endometrium of oestrogen and progesterone receptor, heat shock protein 27, and human beta-lactoglobulin. Hum Reprod 2000, 15:36-42

38. Bjornsdottir LT, Geirsson RT, Jonsson PV: Urinary incontinence and urinary tract infections in octogenarian women. Acta Obstet Gynecol Scand 1998, 77:105-109

39. Kenton K, Sadowski D, Shott S, Brubaker L: A comparison of women with primary and recurrent pelvic prolapse. $\mathrm{Am} J \mathrm{Ob}$ stet Gynecol 1999, 180:1415-1418
Publish with BioMed Central and every scientist can read your work free of charge

"BioMedcentral will be the most significant development for disseminating the results of biomedical research in our lifetime." Paul Nurse, Director-General, Imperial Cancer Research Fund

Publish with BMC and your research papers will be:

- available free of charge to the entire biomedical community

- peer reviewed and published immediately upon acceptance

- cited in PubMed and archived on PubMed Central

- yours - you keep the copyright

Submit your manuscript here:

http://www.biomedcentral.com/manuscript/
BioMedcentral.com ditorial@biomedcentralcom 